\title{
Les relations entre santé et environnement
}

\author{
Jean Martin \\ Dr méd., membre de la rédaction
}

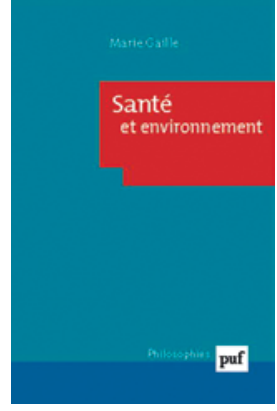

Marie Gaille Santé et environnement

Paris: Presses

Universitaires de France, 2018, 160 pages
Remarque linguistique: "environnement» se dit en allemand Umwelt (ce qui nous entoure) mais il est plus approprié, certains le font, de dire Mitwelt (ce avec quoi nous vivons).
Marie Gaille est philosophe et directrice de recherches au CNRS français. Ses travaux s'intéressent à l'expérience de la maladie et au rapport au milieu de vie. Elle rappelle au début de cet ouvrage une phrase de Claude Bernard: «Il est très difficile, sinon impossible, de poser les limites entre l'état normal et l'état anormal. Les mots santé et maladie sont très arbitraires.»

On sait que, dans le monde occidental, c'est Hippocrate qui a séparé la médecine du religieux - «en combinant une faculté fine d'observation de choses particulières et une capacité à établir des faits généraux». Dans la première partie du livre est décrite l'histoire de cet héritage, dont l'influence a perduré au cours des siècles et au-delà des évolutions épistémologiques. L’attention aux causes externes de la maladie est un des points principaux de cette filiation.

L'auteure a étudié principalement les travaux du psychiatre Kurt Goldstein (1878-1965), pionnier de la neuropsychologie, de Georges Canguilhem et de Maurice Merleau-Ponty; tous philosophes, les deux premiers étant aussi médecins. Gaille souligne: «La réflexion de ces trois auteurs débouche sur une définition de la santé qui a pour axe central la relation à un milieu de vie. La santé n'est pas seulement l'absence de maladie, elle relève de l'expression de capacités, une forme d'aisance.» Elle cite Canguilhem pour lequel «la 'normalité' pour le vivant est relative à l'environnement de celui-ci et n'a pas de signification en dehors de cette relation.» Il importe donc de considérer et de combiner deux approches de la santé: une conception statistique et biologique d'une part, une conception holistique ou humaniste, centrée sur la personne, d'autre part.

Pour Goldstein, la mission de la médecine est «d'aider le patient à retrouver une place dans le monde et une relation à autrui, à se tailler un environnement de vie vivable, dans laquelle son existence a du sens et de la valeur.» On peut encore noter que, pour le psychiatre, la médecine a des dimensions et des implications sociales et politiques majeures - bien admises et démontrées aujourd'hui, même si peu de pays en tirent les conséquences.

Antérieurement, Marie Gaille fait référence aux travaux du Français Pierre-Jean Cabanis à la fin du XVIII siècle. Par exemple, ce dernier assimile la criminalité à une maladie - raisonnement moderne quand on sait l'importance des influences du milieu, dès l'enfance, dans les délinquances comme pour la santé. Cabanis met l'accent sur les effets salutogénétiques d'un milieu naturel et architectural de qualité.

L'auteure présente une importante réflexion sur la notion de handicap, qui n'est pas caractérisée simplement par la déficience d'une personne, mais comme le résultat d'une déficience et d'un environnement qui en aggrave les effets. L'anthropologue québécois Fougeyrollas renvoie à une «organisation sociale qui produit des situations systémiques de discrimination et de stigmatisation - ou encore de privilèges et de pouvoir»! C'est pourquoi il convient aujourd'hui de parler de «situation de handicap». Comme d'autres, Goldstein relève qu'il est possible de se sentir en bonne santé même si, à cause de limitations, on ne peut plus faire certaines choses comme auparavant. Dans ce sens, on peut dire qu'une bonne santé exprime une marge «de tolérance et de compensation des agressions de l'environnement».

Gaille souligne le passage nécessaire de la notion d'interdépendance entre les composantes de la biosphère, beaucoup évoquées à propos du dérèglement clima-

\section{Cet ouvrage est une contribution bienvenue alors que l'importance des humanités en médecine est mieux reconnue.}

tique, à celle de solidarité au sein d'une société où tous partagent la même communauté de destin. Ce qui renvoie à l'accent mis aujourd'hui sur le fait que les actions en matière de climat (vers une justice climatique) doivent être menées en concordance avec des actions d'ordre social.

Santé et environnement est un ouvrage maniable et agréable à lire, très bien informé. Il examine son sujet* du point de vue des sciences humaines et sociales, avec une perspective historique dès Hippocrate. Il apporte une contribution bienvenue alors que l'importance des humanités en médecine (Medical Humanities) est de mieux en mieux reconnue - et nécessaire pour une médecine du XXI ${ }^{\mathrm{e}}$ siècle qui aille au-delà du quantitatif et du technique. 